PROCEEDINGS OF THE

AMERICAN MATHEMATICAL SOCIETY

Volume 136, Number 9, September 2008, Pages 3163-3169

S 0002-9939(08)09495-1

Article electronically published on April 25, 2008

\title{
DENSE-LINEABILITY IN SPACES OF CONTINUOUS FUNCTIONS
}

\author{
L. BERNAL-GONZÁLEZ
}

(Communicated by N. Tomczak-Jaegermann)

\begin{abstract}
In this paper we provide a general method to prove that certain nonlinear families of continuous functions contain dense linear manifolds. An application is furnished.
\end{abstract}

\section{INTRODUCTION}

The study of a rich algebraic structure for a family of functions not being a linear space has recently attracted the attention of an increasing number of analysts. Often, it turns out that although giving a concrete example of a function with a special property can be difficult, we surprisingly obtain that there exist many such functions. This is well-known if we restrict ourselves to obtaining a topologically large set of such functions, by mainly using a Baire-category approach (see [17]). What is not so well-known is that, in many cases, such special functions can even be organized in a linear way. For this, see for instance [1, 2], 9] and the references contained in it.

In order to fix the goal of this paper, we need some recent terminology, which can be found in 12, 3, 1 and 7. Assume that $X$ is a topological vector space and that $M$ is a subset of $X$. Then $M$ is said to be

- lineable if $M \cup\{0\}$ contains an infinite dimensional linear submanifold,

- dense-lineable or algebraically generic if $M \cup\{0\}$ contains a dense linear submanifold,

- spaceable if $M \cup\{0\}$ contains an infinite dimensional closed linear submanifold.

It is clear that "spaceable" implies "lineable" and that, if $X$ is infinite dimensional, "dense-lineable" also implies "lineable".

For instance, Gurariy [10] (see also [11]) proved in 1966 that the family $N D$ of (continuous) nowhere differentiable functions on $[0,1]$ is lineable in the space $C[0,1]$ of continuous functions on $[0,1]$. Later, Fonf, Gurariy and Kadec [8] proved that

Received by the editors June 19, 2007.

2000 Mathematics Subject Classification. Primary 46E10; Secondary 26A16, 26A27, 26E10.

Key words and phrases. Dense-lineability, algebra of functions, continuous nondifferentiable functions, nowhere analytic functions, Hölder functions.

The author has been partially supported by the Plan Andaluz de Investigación de la Junta de Andalucía FQM-127, and by MEC Grants MTM2006-13997-C02-01 and Acción Especial MTM2004-21420-E.

(C)2008 American Mathematical Society Reverts to public domain 28 years from publication 
$N D$ is spaceable in $C[0,1]$, endowed with the supremum norm. In fact, RodríguezPiazza [18] showed that every separable infinite dimensional Banach space is isometrically isomorphic to a linear manifold whose nonzero elements belong to $N D$. Hencl 13 improved this result in 2000 by replacing $N D$ by the class $N H$ of nowhere Hölder functions; in particular, $N H$ is spaceable in $C[0,1]$. In 2005, Bayart [3, pp. 168-169] (see also [2]) demonstrated that, for a prefixed set $E \subset \mathbb{T}(:=$ the unit circle) of Lebesgue measure 0 , the subset of functions in $C(\mathbb{T})$ whose Fourier series expansion diverges at every point of $E$ is dense-lineable.

In this paper we will prove two rather general assertions which are useful to discover dense-lineability, mainly in the context of spaces of continuous functions; see Section 2. In Section 3, an application of such assertions to three families in the space $C[0,1]$ is given. In particular, we complete the aforementioned results by Gurariy and Hencl.

\section{The MAIN RESUlTS}

Our setting will be the vector space $F(T, \mathbb{K})$ of $\mathbb{K}$-valued functions on a topological vector space $T$ or its subspace $C(T, \mathbb{K})$ of corresponding continuous functions. As usual, $\mathbb{K}$ denotes one of the fields $\mathbb{R}$ (the real line) or $\mathbb{C}$ (the complex plane).

Let us fix some additional terminology. A linear manifold $A \subset F(T, \mathbb{K})$ is said to be an algebra if it is stable under multiplication. If, in addition, it satisfies that $1 / f \in A$ whenever $f \in A$ and $f(t) \neq 0$ for all $t \in T$, then we say that $A$ is an algebra with division. A family $\{A(t)\}_{t \in T}$ of subsets of $F(T, \mathbb{K})$ is called locally defined if it satisfies the following property: If $t_{0} \in T, f \in A\left(t_{0}\right), g \in F(T, \mathbb{K})$ and $g=f$ on some neighborhood of $t_{0}$, then $g \in A\left(t_{0}\right)$. Moreover, a family $\{A(t)\}_{t \in T}$ of subsets of $F(T, \mathbb{K})$ is said to be openly defined provided that

$$
A(t)=\bigcup_{U \in O(t)} \bigcap_{\tau \in U} A(\tau) \quad(t \in T)
$$

where $O(t)$ is the collection of all open subsets of $T$ containing $t$.

For instance, if $T=[0,1]$ and we denote $A(t)=\{$ functions $f:[0,1] \rightarrow \mathbb{K}$ that are differentiable at $t\}$ and $\widetilde{A}(t)=\{$ functions $f:[0,1] \rightarrow \mathbb{K}$ that are analytic at $t\}$, then $A(t), \widetilde{A}(t)$ are algebras with division for all $t \in[0,1]$, and both families $\{A(t)\}_{t \in T},\{\widetilde{A}(t)\}_{t \in T}$ are locally defined. Moreover $\{\widetilde{A}(t)\}_{t \in T}$ is openly defined (recall that a function is analytic at a point $t_{0}$ if and only if it is analytic at a neighborhood of $\left.t_{0}\right)$. But $\{A(t)\}_{t \in T}$ is not openly defined; indeed, according to the well-known Weierstrass example (see e.g. [20, pp. 48-49]), the function

$$
f(x):=x^{2} \sum_{n=0}^{\infty} 2^{-n} \sin \left(2 \pi(8 \pi+3)^{n} x\right)
$$

is (continuous on $[0,1]$ but) differentiable only at the origin.

Finally, we establish the two promised assertions about algebraic genericity. The first one is abstract, and its main usefulness is to deduce dense-lineability from the mere lineability. The second one deals with families of continuous functions.

Theorem 2.1. Assume that $X$ is a metrizable separable topological vector space. Suppose that $\Gamma$ is a family of linear submanifolds of $X$ such that $\bigcap_{S \in \Gamma} S$ is dense in $X$ and $\bigcap_{S \in \Gamma}(X \backslash S)$ is lineable. Then $\bigcap_{S \in \Gamma}(X \backslash S)$ is dense-lineable. 
Proof. From the hypothesis, we can choose a dense countable set $\left\{z_{n}\right\}_{n \geq 1}$ in $X$ as well as a translation-invariant distance $d$ defining the topology of $X$. By denseness, we also can take, for each $\mathbb{N}:=\{1,2, \ldots\}$, a vector $y_{n} \in \bigcap_{S \in \Gamma} S$ such that

$$
d\left(y_{n}, z_{n}\right)<1 / n \text {. }
$$

Since $\bigcap_{S \in \Gamma}(X \backslash S)$ is lineable, there is an infinite dimensional linear manifold $L$ with

$$
L \backslash\{0\} \subset \bigcap_{S \in \Gamma}(X \backslash S) .
$$

Therefore we can select a linearly independent sequence of vectors $\left\{v_{n}\right\}_{n \geq 1} \subset L$. Since scalar multiplication is continuous on $X$, we derive the existence of a sequence $\left\{\varepsilon_{n}\right\}_{n \geq 1} \subset(0,+\infty)$ such that

$$
d\left(\varepsilon_{n} v_{n}, 0\right)<1 / n \quad(n \in \mathbb{N}) .
$$

Now, we define $x_{n}:=y_{n}+\varepsilon_{n} v_{n}(n \geq 1)$ and

$$
D:=\operatorname{span}\left(\left\{x_{n}: n \geq 1\right\}\right) \text {. }
$$

Thanks to (1), (2), the triangle inequality and the translation-invariance of $d$, we get

$$
\begin{aligned}
d\left(x_{n}, z_{n}\right) & \leq d\left(y_{n}+\varepsilon_{n} v_{n}, y_{n}\right)+d\left(y_{n}, z_{n}\right) \\
& =d\left(\varepsilon_{n} v_{n}, 0\right)+d\left(y_{n}, z_{n}\right)<2 / n \longrightarrow 0(n \rightarrow \infty) .
\end{aligned}
$$

From (3) and the denseness of $\left\{z_{n}\right\}_{n \geq 1}$, we derive that $\left\{x_{n}\right\}_{n \geq 1}$ is dense. Consequently, $D$ is a dense linear submanifold of $X$.

It remains to prove that $D \backslash\{0\} \subset \bigcap_{S \in \Gamma}(X \backslash S)$. To this end, fix a vector $x \in D \backslash\{0\}$. Then there exist $N \in \mathbb{N}$ and scalars $c_{1}, \ldots, c_{N}$ with $c_{N} \neq 0$ satisfying $x=c_{1} x_{1}+\cdots+c_{N} x_{N}$; that is,

$$
x=c_{1} y_{1}+\cdots+c_{N} y_{N}+c_{1} \varepsilon_{1} v_{1}+\cdots+c_{N} \varepsilon_{N} v_{N} .
$$

Assume, by way of contradiction, that $x \notin \bigcap_{S \in \Gamma}(X \backslash S)$. Then there would be some $S_{0} \in \Gamma$ for which $x \in S_{0}$. But $y_{1}, \ldots, y_{N} \in \bigcap_{S \in \Gamma} S \subset S_{0}$ and $S_{0}$ is a linear manifold, so

$$
x-\left(c_{1} y_{1}+\cdots+c_{N} y_{N}\right) \in S_{0} .
$$

Since $c_{N} \varepsilon_{N} \neq 0$ and the vectors $v_{n}$ are linearly independent, we deduce that $c_{1} \varepsilon_{1} v_{1}+$ $\cdots+c_{N} \varepsilon_{N} v_{N} \in L \backslash\{0\} \subset \bigcap_{S \in \Gamma}(X \backslash S)$, which contradicts (5) because of (4).

Theorem 2.2. Let $T$ be a topological space and let $X$ be a topological vector space of $\mathbb{K}$-valued continuous functions on $T$ that is an algebra. Assume that $\{A(t)\}_{t \in T}$ is a family of subsets of $F(T, \mathbb{K})$, and denote $A:=\bigcap_{t \in T}(A(t) \cap X), B:=\bigcap_{t \in T}(X \backslash A(t))$.

(A) Suppose that the following conditions are satisfied:

(a) For any $t \in T$, the set $A(t)$ is an algebra with division.

(b) The family $\{A(t)\}_{t \in T}$ is locally defined and openly defined.

(c) B is not empty.

(d) There exists a function $\varphi \in A$ such that the image $\varphi(U)$ of every nonempty open subset $U \subset T$ is an infinite set.

Then the set $B$ is lineable.

(B) If, in addition, $X$ is metrizable and separable and $A$ is dense in $X$, then $B$ is dense-lineable in $X$. 
Proof. Part (B) follows from (A) and Theorem 2.1 as applied to $\Gamma:=\{A(t) \cap X\}_{t \in T}$. In order to demonstrate (A), let us assume that conditions (a) to (d) hold. Consider the function $\varphi$ furnished by (d). By using (c), we can define the set

$$
D:=\{(P \circ \varphi) F: P \in \Lambda\},
$$

where $\Lambda$ is the family of all polynomials $P: \mathbb{K} \rightarrow \mathbb{K}$ and $F \in B$. Then $D$ is, evidently, a linear submanifold of $X$. In order to see that $\operatorname{dim}(D)=\infty$, it is enough to show that, for each $N \in \mathbb{N}$, the functions $F, \varphi F, \ldots, \varphi^{N} F$ (which are in $D$ ) are linearly independent. This is true because, otherwise, there would be a nonzero polynomial $P$ such that $(P \circ \varphi) F=0$. By (a), the set $A$ is an algebra, so $P \circ \varphi \in A$. Taking $U=T$ in $(\mathrm{d})$, we have that $\varphi(T)$ is infinite, so we can select a point $t_{0} \in T$ such that $P\left(\varphi\left(t_{0}\right)\right) \neq 0$; indeed, $P$ has only finitely many zeros. By continuity, $P(\varphi(t)) \neq 0$ for all $t$ belonging to some neighborhood of $t_{0}$. Hence $F(t)=0$ on such a neighborhood, which by (b) implies that $F \in A\left(t_{0}\right)$, a contradiction. Thus, $\operatorname{dim}(D)=\infty$.

It remains to prove that $D \backslash\{0\} \subset B$. For this, fix a function $f \in D \backslash\{0\}$. Then there is a nonzero polynomial $P$ with $f=(P \circ \varphi) F$. Assume, by way of contradiction, that $f \notin B$. Then there exists a point $t_{0} \in T$ such that $f \in A\left(t_{0}\right)$. Since $\{A(t)\}_{t \in T}$ is openly defined, we can find an open set $U \subset T$ with $t_{0} \in U$ such that $f \in A(t)$ for all $t \in U$. Due to (d), the set $\varphi(U)$ is infinite, so one can choose a point $t_{1} \in U$ such that $P\left(\varphi\left(t_{1}\right)\right) \neq 0$. Again by continuity, there is an open set $U_{1}$ with $t_{1} \in U_{1} \subset U$ satisfying $P(\varphi(t)) \neq 0$ for all $t \in U_{1}$. Let us consider the function $\psi: X \rightarrow \mathbb{K}$ given by

$$
\psi(t)= \begin{cases}\frac{f(t)}{P(\varphi(t))} & \text { if } t \in U_{1}, \\ 1 & \text { if } t \in X \backslash U_{1} .\end{cases}
$$

Since $A\left(t_{1}\right)$ is a locally defined algebra with division, we obtain (observe that $f$ and $P \circ \varphi$ are in $\left.A\left(t_{1}\right)\right)$ that $\psi \in A\left(t_{1}\right)$. But $F=f /(P \circ \varphi)$ on $U_{1}$. Thus, once more by local definition, our function $F$ belongs to $A\left(t_{1}\right)$, which is absurd.

Remark 2.3. In view of the last theorem, one is tempted to say that if there are sufficiently many "good" functions (the set $A$ ) enjoying an adequate structure, then the set of "pathological" functions (the set $B$ ) is either empty or algebraically huge.

\section{An APplication}

In this section, we apply the statements obtained in Section 2 to three families within the space of continuous functions on $[0,1]$. Let us recall some standard notation and facts. We set $\mathbb{N}_{0}:=\mathbb{N} \cup\{0\}$ and $f^{(0)}:=f$ for any function $f$. If $p \in \mathbb{N}_{0}$, then the algebra $C^{p}[0,1]$ of $C^{p}$-functions $f:[0,1] \rightarrow \mathbb{K}$ becomes a

Banach space when endowed with the norm $\|f\|:=\sum_{j=0}^{p} \sup _{[0,1]}\left|f^{(j)}\right|$ (see [14]). This norm generates the topology of uniform convergence of functions and their derivatives up to the order $p$. Moreover, the algebra $C^{\infty}[0,1]$ of infinitely many times differentiable functions on $[0,1]$ can be endowed with a distance $d$ generating the topology of uniform convergence of derivatives of all orders. For instance, we can take $d(f, g)=\sum_{j=0}^{\infty} 2^{-j} \min \left\{1, \sup _{[0,1]}\left|f^{(j)}\right|\right\}$. So all spaces $C^{p}[0,1]\left(p \in \mathbb{N}_{0} \cup\{\infty\}\right)$ are metrizable and complete. They are also separable: the set of polynomials is dense in each of them.

By using a Baire category approach, it has been proved that the family of continuous functions on $[0,1]$ that are nowhere differentiable is not also nonempty but 
is even residual in $C[0,1]$ [17, pp. 45-46] (recall that a residual set $A$ in a Baire space is a subset whose complement is of the first category; in some sense, $A$ is topologically large). Also the family of $C^{\infty}$-functions on $[0,1]$ that are nowhere analytic is residual in $C^{\infty}[0,1]$ (see [16], [19] and [5]). By applying the results of the last section, we will be able to prove that both families are also algebraically large. Note that Proposition 3.1 below improves Gurariy's result [10.

Proposition 3.1. Let $p \in \mathbb{N}_{0}$. Then the class of functions $f \in C^{p}[0,1]$ such that $f^{(p)}$ is nowhere differentiable on $[0,1]$ is dense-lineable in $C^{p}[0,1]$.

Proof. Let us denote by $M(p)$ the class of the statement of the theorem. For the case $p=0$, we know by [10] that $M(0)$ is lineable. Thus, it is enough to apply Theorem 2.1 to $X=C[0,1]$ and $\Gamma=\{S(t)\}_{t \in[0,1]}$, where $S(t)=\{f \in$ $X: f$ is differentiable at $t\}$. Finally, recall that the polynomials are differentiable everywhere and form a dense set.

Assume now that $p \in \mathbb{N}$. As expected, this time we apply Theorem 2.1 to $X=C^{p}[0,1]$ and $\Gamma=\{S(t)\}_{t \in[0,1]}$, where $S(t)=\left\{f \in X: f^{(p)}\right.$ is differentiable at $t\}$. Again, recall that the polynomials are $p+1$ times differentiable everywhere and form a dense subset of $C^{p}[0,1]$. So it must be proved that the class $M(p)$ is lineable. To see this, consider a linearly independent sequence $\left\{f_{n}\right\}_{n \geq 1}$ whose linear span is included in $M(0) \cup\{0\}$ and, for each $n \in \mathbb{N}$, denote by $F_{n}$ the unique antiderivative of order $p$ of $f_{n}$ such that $F_{n}^{(k)}(0)=0$ for all $k=0, \ldots, p-1$. It is clear that $\operatorname{span}\left(\left\{F_{n}\right\}_{n \geq 1}\right) \subset M(p) \cup\{0\}$. Now if $c_{1}, \ldots, c_{N}$ are scalars satisfying $c_{1} F_{1}+\cdots+c_{N} F_{N}=0$ on $[0,1]$, then after $p$ derivations we are driven to $c_{1} f_{1}+$ $\cdots+c_{N} f_{N}=0$ on $[0,1]$, which implies $c_{1}=\cdots=c_{N}=0$. Therefore the sequence $\left\{F_{n}\right\}_{n \geq 1}$ is linearly independent and $M(p)$ is lineable.

Proposition 3.2. The class of functions $f \in C^{\infty}[0,1]$ which are nowhere analytic on $[0,1]$ is dense-lineable in $C^{\infty}[0,1]$.

Proof. The existence of nowhere analytic $C^{\infty}$-functions is known at least from $\mathrm{du}$ Bois-Reymond [6. A specially elegant easy example is that of Lerch [15]:

$$
f(t)=\sum_{n=1}^{\infty} \frac{\cos \left(3^{n} t\right)}{n !} .
$$

Then it is sufficient to apply Theorem 2.2 with $T=[0,1], X=C^{\infty}[0,1], A(t)=$ $\{f \in F([0,1], \mathbb{K}): f$ is analytic at $t\}$ and $\varphi(t)=t(t \in[0,1])$. Take into account that the polynomials are in the corresponding set $A$.

Finally, let us denote by $C B V[0,1]$ the algebra of all continuous functions on $[0,1]$ with bounded variation. It is well-known that it becomes a Banach space under the norm $\|f\|:=\sup _{[0,1]}|f|+V(f)$, where $V(f)$ is the variation of $f$ on $[0,1]$; that is, $V(f):=\sup \left\{\sum_{j=1}^{N}\left|f\left(t_{j}\right)-f\left(t_{j-1}\right)\right|: 0=t_{0}<t_{1}<t_{2}<\cdots<t_{N}=1, N \in \mathbb{N}\right\}$. Then $C B V[0,1]$ is a metrizable space. Moreover, it is separable, because the set of polynomials is also dense in this space; indeed, $C^{1}[0,1]$ contains the polynomials and is continuously contained in $C B V[0,1]$. Our final assertion has to do with functions in this space which are not differentiable at any interval.

Proposition 3.3. The class of functions $f \in C B V[0,1]$ such that $f$ is differentiable on no interval in $[0,1]$ is dense-lineable in $C B V[0,1]$. 
Proof. Just apply Theorem 2.2 to $X=C B V[0,1], A(t)=\{f \in F([0,1], \mathbb{K}): f$ is differentiable on some neighborhood of $t\}$ and $\varphi(t)=t(t \in[0,1])$. Again, the corresponding set $A$ contains the polynomials, so it is dense. It remains to show that the class of the statement is not empty. This is a consequence of a more general result; namely, given a set $E \subset[0,1]$ with Lebesgue measure 0 , there is an increasing (so of bounded variation) continuous function on $[0,1]$ which is differentiable at no point of $E$ (see [20, pp. 25-26]). Now it is enough to choose $E=\{$ the rational points in $[0,1]\}$.

Remarks 3.4. 1. Assume that $\varphi:[0,+\infty) \rightarrow[0,+\infty)$ is a continuous strictly increasing function with $\varphi(0)=0$. Then a function $f:[0,1] \rightarrow \mathbb{K}$ is said to be $\varphi$-Hölder (or $\varphi$-Lipschitz) at a point $x_{0} \in[0,1]$ whenever there are a neighborhood $U \subset[0,1]$ of $x_{0}$ and a constant $M=M\left(f, x_{0}\right) \in(0,+\infty)$ such that

$$
\left|f(x)-f\left(x_{0}\right)\right| \leq M \varphi\left(\left|x-x_{0}\right|\right) \text { for all } x \in U .
$$

If, in addition, $f \in C[0,1]$, then one can take $U=[0,1]$. By Hencl's result [13, the family of functions $f \in C[0,1]$ which are nowhere $\varphi$-Hölder is spaceable, and so is lineable. Hence, with a very similar proof, Proposition 3.1 can be reinforced by changing "nowhere differentiable" to "nowhere $\varphi$-Hölder". This complements Fonf-Gurariy-Kadec-Rodríguez-Hencl's statements about spaceability.

2. With a different proof, we have obtained in 4] a statement that is stronger than Proposition 3.2. Namely, the class of functions $f \in C^{\infty}[0,1]$ such that the associated Taylor series has null radius of convergence at each point is dense-lineable.

3. In Proposition 3.3 one cannot replace the condition "differentiable on no interval" by "nowhere differentiable". Indeed, every function of bounded variation is differentiable almost everywhere.

\section{REFERENCES}

[1] R.M. Aron, V.I. Gurariy and J.B. Seoane, Lineability and spaceability of sets of functions on $\mathbb{R}$, Proc. Amer. Math. Soc. 133 (2005), 795-803. MR.2113929 (2006i:26004)

[2] R. Aron, D. Pérez-García and J.B. Seoane-Sepúlveda, Algebrability of the set of nonconvergent Fourier series, Studia Math. 175 (2006), 83-90. MR2261701 (2007k:42007)

[3] F. Bayart, Topological and algebraic genericity of divergence and universality, Studia Math. 167 (2005), 161-181. MR2134382 (2006b:46024)

[4] L. Bernal-González, Lineability of sets of nowhere analytic functions, J. Math. Anal. Appl. 340 (2008), 1284-1295.

[5] R.B. Darst, Most infinitely differentiable functions are nowhere analytic, Canad. Math. Bull. 16 (1973), 597-598. MR0346112 (49:10838)

[6] P. du Bois-Reymond, Uber den Gültigkeitsbereich der Taylor'schen Reihenentwicklung, Math. Ann. 21 (1883), 109-117. MR 1510189

[7] P. Enflo and V.I. Gurariy, On lineability and spaceability of sets in function spaces, preprint.

[8] V.P. Fonf, V.I. Gurariy and M.I. Kadec, An infinite dimensional subspace of $C[0,1]$ consisting of nowhere differentiable functions, C.R. Acad. Bulgare Sci. 52 (1999), nos. 11-12, 13-16. MR.1738120 (2000j:26006)

[9] F.J. García-Pacheco, N. Palmberg and J.B. Seoane-Sepúlveda, Lineability and algebrability of pathological phenomena in analysis, J. Math. Anal. Appl. 326 (2007), 929-939. MR.2280953 (2007i:26003)

[10] V.I. Gurariy, Subspaces and bases in spaces of continuous functions (Russian), Dokl. Akad. Nauk SSSR 167 (1966), 971-973. MR0199674 (33:7817)

[11] V.I. Gurariy, Linear spaces composed of everywhere nondifferentiable functions, C.R. Acad. Bulgare Sci. 44 (1991), no. 5, 13-16. MR.1127022 (92j:46046)

[12] V. Gurariy and L. Quarta, On lineability of sets of continuous functions, J. Math. Anal. Appl. 294 (2004), 62-72. MR2059788 (2005c:46026) 
[13] S. Hencl, Isometrical embeddings of separable Banach spaces into the set of nowhere approximately differentiable and nowhere Hölder functions, Proc. Amer. Math. Soc. 128 (2000), 3505-3511. MR 1707147 (2001b:46026)

[14] J. Horváth, "Topological Vector Spaces and Distributions", Vol. 1, Addison-Wesley, Reading, MA, 1966. MR0205028 (34:4863)

[15] M. Lerch, Ueber die Nichtdifferentiirbarkeit bewisser Funktionen, J. Reine Angew. Math. 103 (1888), 126-138.

[16] D. Morgenstern, Unendlich oft differenzierbare nicht-analytische Funktionen, Math. Nachr. 12 (1954), 74. MR0064830(16:342b)

[17] J.C. Oxtoby, "Measure and Category", Springer, Berlin, 1980. MR584443 (81j:28003)

[18] L. Rodríguez-Piazza, Every separable Banach space is isometric to a space of continuous nowhere differentiable functions, Proc. Amer. Math. Soc. 123 (1995), 3649-3654. MR1328375 (96d:46007)

[19] H. Salzmann and K. Zeller, Singularitäten unendlicht oft differenzierbarer Funktionen, Math. Z. 62 (1955), 354-367. MR0071479 (17:134b)

[20] A.C.M. Van Rooij and W.H. Schikhoff, "A Second Course on Real Functions", Cambridge University Press, Cambridge, 1982.

Facultad de Matemáticas, Departamento de Análisis Matemático, Universidad de Sevilla, Apdo. 1160, Avda. Reina Mercedes, Sevilla-41080, Spain

E-mail address: lbernal@us.es 